cancer - the diameter of the tumour and the number of lymph nodes containing cancerous cells, which indicates whether the primary disease had spread at the start of treatment. However, even among women with small, node-negative tumours, the risk of metastasis was about $10 \%$ over the 15 -year period.

Pan et al. acknowledge that there are several caveats to their analysis, including a lack of available data on how many women completed their adjuvant treatment, and suboptimal treatment of people who had one particular type of ER-positive breast cancer, dubbed HER2-positive. Nonetheless, it is clear that, even after adjuvant endocrine therapy, women with ER-positive, early-stage breast cancer remain at persistent risk of recurrence for at least 20 years after the original diagnosis. These findings, together with data from another analysis of breast-cancer relapse ${ }^{2}$, might have implications for long-term followup strategies, and possibly for treatments.

One way to decrease the risk of relapse might be to extend the duration of adjuvant endocrine therapy - a strategy that is already being tested. We wonder, however, whether this approach will be sufficient to reduce or avoid the risk of late metastasis. One trial ${ }^{3}$ published in 2016 indicates that increasing the duration of adjuvant therapy from five to ten years significantly improves rates of disease-free survival five years after the treatment ceases, and lowers the incidence of cancer arising in the previously unaffected breast - but does not increase overall survival rates. A second ${ }^{4}$ trial compared continuous adjuvant therapy between years five and ten with an 'on-off' therapy that aimed to resensitize cancer cells that might have become resistant to the therapy. It found no difference in rates of metastasis-free survival on completion of either treatment.

Longer follow-ups are needed to better understand the effects of extended adjuvant endocrine therapy, because of the slowly progressive nature of the disease. Nonetheless, it is clear that, although extending the duration of therapy can play a part in preventing late relapses, it might well need to be given for the rest of a woman's life to be effective. This would raise issues of toxicity, compliance and cost.

To best determine other possible ways to reduce the risk of relapse, we must consider what might cause the dormant tumour cells from which metastasis arises to reawaken after many years. Dormant cells have escaped destruction by the body's immune system and entered a microenvironment that supports their survival ${ }^{5}$. Once in this niche, many mechanisms might underlie dormancy — indeed, dormancy can be thought of as a multidimensional status, which involves multiple factors (Fig. 1).

One dimension is cellular dormancy, in which intrinsic or extrinsic factors drive cells into a resting state. Intrinsic factors might include changes that reduce the cell's drive to divide, similar to those that limit proliferation in cancer stem cells (for example, see ref. 6). These changes could be epigenetic - modulating gene expression without affecting the underlying DNA sequence - or genetic ${ }^{5}$. Extrinsic factors include crosstalk between different cell types in the surrounding microenvironment, such as endothelial cells that line vessels, immune cells and fibroblasts, which make up the structural framework of tissues. A second dimension is vasculature-related dormancy, in which the tumour-cell population is kept small because poor vascularization in the region leads to a lack of nutrients and oxygen. A third dimension is immune-mediated dormancy, in which the immune system controls an expanding tumour-cell population by continuously searching for and eliminating cancerous cells.

If this dormancy status becomes unbalanced owing to changes in any of these factors, dormant cells awaken and metastatic disease develops. Thus, strategies to prevent relapse should aim either to stop dormant cells from awakening or to destroy them when dormant. To achieve this goal, it would be beneficial to identify those patients who will have a late relapse and to tailor a therapeutic strategy to them. ER-positive breast cancers have a low level of intratumoral cell diversity ${ }^{7}$, such as distinct forms of genetic, epigenetic and functional diversity. A better understanding of this diversity might lead to the identification of factors that enable certain cells to become resistant to endocrine therapy and survive in metastatic niches.

Perhaps the major effect of extended adjuvant endocrine therapy is to keep tumour cells dormant for a longer, but still temporary, period of time. We believe that a combined strategy that concomitantly targets both the tumour cells and the surrounding microenvironment has a higher probability of destroying the dormant cells or of inducing a lifelong state of dormancy, and hence provides a higher probability of a cure. A better understanding of the crosstalk between the dormant cells, their surrounding cell types and the immune system is crucial for developing effective microenvironment-directed therapies. In addition, attention should be paid to the possibility that dormant breast tumour cells undergo evolutionarily conserved programs that lead to a stem-cell-like, prolonged resting state. Maintaining this stem-cell status permanently might be another way to prevent dormant cells from reawakening.

Giuseppe Curigliano is in the Department of Hematology and Oncology, European Institute of Oncology, University of Milan, 20141 Milan, Italy. Fatima Cardoso is at the Breast Unit, Champalimaud Clinical Center, Champalimaud Foundation, 1400-038

Lisbon, Portugal.

e-mails: giuseppe.curigliano@ieo.it; fatimacardoso@fundacaochampalimaud.pt

1. Pan, H. et al. N. Engl. J. Med. 377, 1836-1846 (2017).

2. Colleoni, M. et al. J. Clin. Oncol. 34, 927-935 (2016).

3. Goss, P. E. et al. N. Engl. J. Med. 375, 209-219

(2016).

4. Colleoni, M. et al. Lancet Oncol. 19, 127-138 (2017)

5. Goss, P. E. \& Chambers, A. F. Nature Rev. Cancer 10 , 871-877 (2010).

6. Tosoni, D. et al. EMBO Mol. Med. 9, 655-671 (2017).

7. Ellis, M. J. \& Perou, C. M. Cancer Discov. 3, 27-34 (2013).

This article was published online on 29 January 2018.

\title{
Reactive carbon species tamed for synthesis
}

\section{A highly reactive form of carbon, known as a carbyne, holds great promise for organic synthesis, but has been difficult to prepare. Reactions that produce carbyne equivalents now unleash this synthetic potential. SEE LETTER P.86}

\section{ROHAN E. J. BECKWITH}

$\mathrm{T}$ The basis of organic chemistry is the study of carbon-containing compounds with the aim of manipulating carbon atoms to generate new molecules through the formation of carbon-carbon (C-C) bonds. On page 86, Wang et al. ${ }^{1}$ report a method for harnessing a reactive form of carbon known as a carbyne (Fig. 1a), which has been underused in synthetic chemistry. The findings open the door to new types of $\mathrm{C}-\mathrm{C}$ bond-formation reaction. Conventional approaches to $\mathrm{C}-\mathrm{C}$ bond formation typically involve well-studied, reactive carbon species. Carbynes, however, have been largely unexplored for $\mathrm{C}-\mathrm{C}$ bond formation because their high reactivity makes them challenging to prepare and handle. Once formed, carbynes react with each other, with solvent molecules and with other substrates in an uncontrolled manner, producing myriad products. This has limited their applications, 
and even efforts to study these species.

Carbynes have previously been formed as complexes with metals, which can then be decomposed to release the free carbyne (see refs 2 and 3, for example). By conducting such decompositions in water at room temperature, researchers have prepared simple compounds that contain $\mathrm{C}-\mathrm{C}$ triple bonds from the reactions of free carbynes with each other ${ }^{4}$. The formation of undesired side products in these reactions was minimized because the rate of reaction of the free carbynes with water was several times slower than the rate of the carbyne-carbyne reaction ${ }^{5}$. However, product yields were low, and the method has limited applications for synthesis.

Wang et al. have devised a clever means of accessing equivalents of carbynes, an approach that has broad synthetic utility. The authors prepared stable precursor compounds that contain two 'masking' groups (Fig. 1b). These precursors can be activated by a catalyst in a light-mediated process so that one of the masks is released, generating a carbyne equivalent. Further activation allows the carbyne equivalent to react with substrate molecules, losing the second mask and forming three new bonds in a single synthetic step.

The authors demonstrated the power of this single-step approach by transforming isobutylbenzene (a simple hydrocarbon that contains a benzene ring) into a more-complex system, installing a new ring in a process that forms two new $\mathrm{C}-\mathrm{C}$ bonds and one carbon-hydrogen $(\mathrm{C}-\mathrm{H})$ bond (Fig. 1c). The reaction occurs with high selectivity at particular carbon atoms on isobutylbenzene, and under conditions that chemists would describe as very mild: at ambient temperature and pressure, and using reagents that tolerate the presence of a wide range of groups in the substrate molecule. Such conditions avoid unwanted degradation of the starting materials or the product, thus maximizing the potential product yield.

Wang and colleagues also showed that the unmasking process could be conducted in a stepwise manner by modifying the reaction conditions, allowing the isolation of compounds in which groups known as diazoacetates are attached to benzene rings; one of the masks is retained as part of the diazoacetate group (Fig. 1d). Although methods for making diazoacetates attached to benzene rings have been reported previously, they require a synthetic 'handle' - a reactive atom or group - to be present on a benzene ring in the starting material ${ }^{6}$. The authors' method installs diazoacetates directly at a $\mathrm{C}-\mathrm{H}$ bond on a benzene ring, and so does not require a synthetic handle. It might, therefore, allow diazoacetates to be attached to complex aromatic systems (compounds that contain a benzene ring, or a related ring system) into which synthetic handles cannot be incorporated.

The authors show that the diazoacetateinstalling reaction works effectively for a

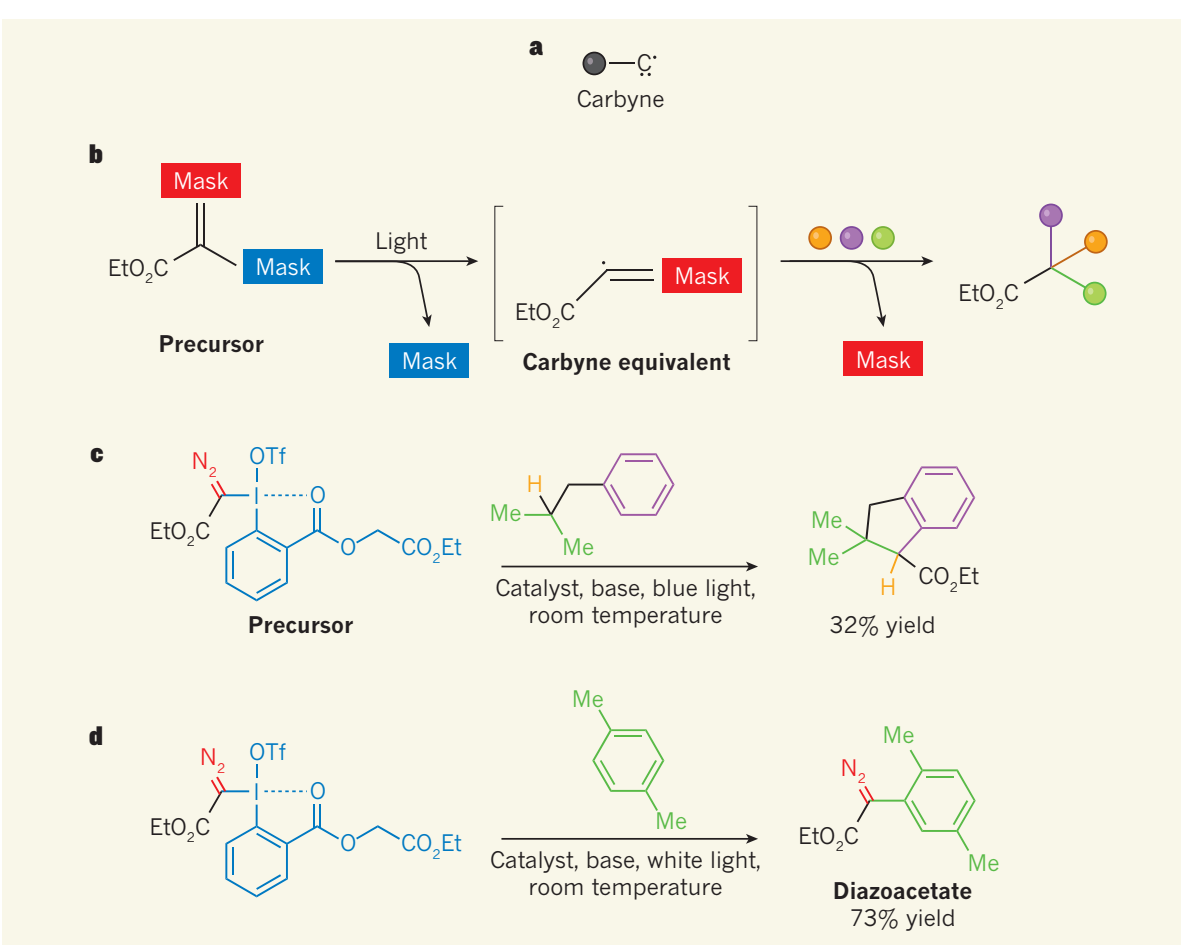

Figure 1 | Carbyne equivalents for synthetic chemistry. a, Carbynes are reactive carbon-containing species with potential uses in organic synthetic chemistry. Dots indicate electrons; black sphere represents any organic chemical group. $\mathbf{b}$, Wang et al. ${ }^{1}$ report a strategy that allows equivalents of carbynes to be prepared from precursor compounds that contain two 'masking' groups. One mask is removed in a lightmediated process, generating the carbyne equivalent; square brackets indicate that the carbyne equivalent is formed transiently. If the equivalent is generated in the presence of substrate molecules (coloured spheres), it reacts to form three new bonds in a single synthetic step, losing the second mask in the process. Et, ethyl group. c, In this colour-coded example, the authors' precursor reacts with three different groups in a single molecule of isobutylbenzene, when irradiated with blue light at room temperature in the presence of a catalyst and a base. OTf, triflate $\left(\mathrm{SO}_{3} \mathrm{CF}_{3}\right) ; \mathrm{Me}$, methyl. d, When white light is used, diazoacetate products form in which one of the masks (a diazo group, red) is retained, as in this example.

range of aromatic substrates. In each case, the diazoacetate is positioned highly selectively at a particular site - even for substrates that contain bulky groups, which often erode site selectivity in other types of reaction. Moreover, the reactions tolerate the presence of a variety of chemical groups in the substrates, and give reasonable yields of products.

The diazoacetate group is especially useful for chemical synthesis because it can be converted into many other groups using metalcatalysed reactions (as Wang et al. demonstrate for diazoacetates made using their approach). The availability of a method that allows diazoacetate groups to be incorporated site selectively into structurally complex molecules will be particularly useful for researchers working in drug discovery, because it will enable variants of biologically active molecules to be made rapidly from the molecules themselves, rather than in multi-step synthetic routes from simple starting materials. Indeed, Wang et al. demonstrate effective, site-selective diazoacetate incorporation into 12 complex drug molecules.

There are some limitations to the new reactions - for example, drug compounds that contain basic amine groups tend not to be amenable to this approach. The yields obtained for diazoacetate incorporation into drug molecules are often low to moderate (10-58\%) compared with the yields observed for the less-complex substrates (mostly 50-99\%). Moreover, substrates that bear strongly electron-withdrawing groups on the aromatic ring also seem to reduce yields and site selectivity relative to other substrates. But these are minor issues compared with the benefits of this approach for drug discovery and development.

Wang and colleagues' work also has more-fundamental implications for organic chemistry. It offers a new means of generating diazo compounds (the family of compounds to which diazoacetates belong) that allows access to types of diazo molecule that were not previously accessible using other chemistries. In addition, because the reactions generate three new bonds, they provide a way to convert simple reagents into structurally complex molecules in a single step. The authors' reactions are therefore an invaluable addition to the synthetic chemist's toolbox.

Finally, Wang et al. have reported the first evidence that carbynes can be harnessed effectively for practical organic synthesis. The findings therefore open the door to the exploration of new reactivities of carbon - either through modification of the currently reported masked 
carbyne equivalents, or through the development of alternative ones.

Rohan E. J. Beckwith is at the Novartis Institutes for BioMedical Research, Inc., Cambridge, Massachusetts 02139, USA. e-mail:rohan.beckwith@novartis.com

1. Wang, Z., Herraiz, A. G., del Hoyo, A. M. \& Suero, M. G. Nature 554, 86-91 (2018).

2. Fischer, E. O., Ruhs, A. \& Plabst, D. Z. Naturforsch. B 32B, 802-804 (1977).

3. Levy, O., Musa, S. \& Bino, A. Dalton Trans. 42,
12248-12251 (2013)

4. Bino, A., Ardon, M. \& Shirman, E. Science $\mathbf{3 0 8 ,}$ 234-235 (2005).

5. Bogoslavsky, B. et al. Angew. Chem. Int. Edn 51, 90-94 (2012)

6. Ye, F. et al. J. Am. Chem. Soc. 137, 4435-4444 (2015).

\section{A non-tailed twist in the viral tale}

\section{Microscopy studies indicate that the most common viruses in the sea lack a tail structure. However, most cultured marine viruses have tails. A family of these elusive non-tailed marine viruses has now been identified. SEE LETTER P.118}

\section{JULIO CESAR IGNACIO-ESPINOZA \& JED A. FUHRMAN}

$\mathrm{B}$ acteriophages, the viruses that infect bacteria, are thought to be the most abundant biological entities on Earth ${ }^{1}$. It has been estimated that, if lined up end to end, bacteriophages from the oceans alone would cover a distance of 3 million parsecs, past many distant galaxies ${ }^{2}$. Most bacteriophages cultured in the laboratory or represented in DNA-sequence databases have a 'tail' structure, which might take the form of a tube with spider-leg-like protrusions (similar in shape to the base of the Apollo Moon lander). However, electron-microscopy analysis of ocean samples indicates that oceanic viruses are predominantly non-tailed ${ }^{3-5}$. Therefore, identifying the 'missing' non-tailed marine viruses might improve our understanding of how viruses regulate the microbial systems that control a large fraction of global carbon and nitrogen cycling ${ }^{2,6}$. Onn page 118, Kauffman et al. ${ }^{7}$ report the discovery of a non-tailed viral family that they suggest might be an important component of the missing viruses, and they explain why this group might have eluded detection until now.

Viruses can be key drivers of the evolution, community composition and mortality of microorganisms, although their major role in the oceans was not recognized until the $1990 \mathrm{~s}^{2,6}$. The number of species a virus can infect is a crucial factor that can influence

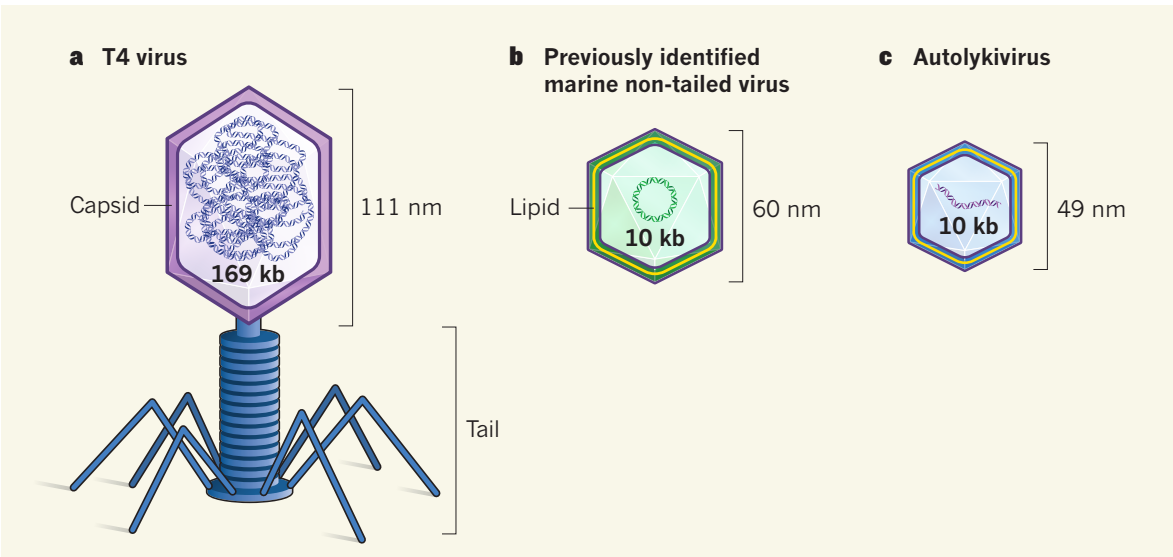

Figure 1 | Different viral forms. Viruses that contain a tail structure are the most common type of bacterium-infecting virus (bacteriophage) cultured in the laboratory or represented in DNA databases. However, in samples taken from marine environments, non-tailed viruses are more common ${ }^{3-5}$. Kauffman et al. ${ }^{7}$ report a previously unknown family of non-tailed marine viruses. a, T4, an example of a tailed virus. Its 169-kilobase genome is enclosed in a capsid structure, made of protein (dark purple), that is 111 nanometres long ${ }^{15}$. The average capsid length for tailed marine viruses ${ }^{5}$ is $65 \mathrm{~nm}$. The tail structures in certain other types of tailed virus have a different shape from that of T4. $\mathbf{b}$, The cortovirus PM2, one of the few non-tailed marine bacteriophages identified so far. PM2 has lipid (yellow) associated with its capsid. Non-tailed marine viruses have an average capsid size $\mathrm{s}^{5} 54 \mathrm{~nm}$. c, An autolykivirus, a member of a family of non-tailed marine viruses identified by Kauffman et al. ${ }^{7}$. The properties of these bacteriophages are consistent with the presence of lipid.

gene transfer between species and the spread of viral infection. Bacteriophage action is usually studied using models in which a virus infects only one or a small group of closely related host strains or species, because this is the pattern observed for most of the bacteriophages cultured so far. And yet such cultures represent just a small proportion ${ }^{8}$ of the hundreds of known bacterial phyla ${ }^{9}$.

Kauffman and colleagues sought to expand the breadth of marine viruses known. They analysed water samples collected off the coast of Massachusetts on three separate days, and attempted to identify bacteriophages that could infect any of the 1,334 strains of Vibrionaceae bacteria that they had isolated. The Vibrionaceae are an easily cultured bacterial group that contains the cholera-causing pathogen Vibrio cholerae, as well as more-benign relatives, such as bioluminescent bacteria that form symbiotic relationships with fishes and squid.

Of all the bacteria that the authors tested, 239 strains became infected with viruses, and Kauffman et al. isolated 241 previously unknown viruses, of which 18 were non-tailed. They named this non-tailed family (Fig. 1) the Autolykiviridae, after Autolykos, an elusive thief from Greek mythology who could not be caught. DNA-sequence analysis revealed that autolykiviruses have small genomes (approximately 10 kilobases in length), which diverge enough from those of known viruses to form their own distinct lineage. They differ from other bacteriophages in sequences encoding a specific structural fold in a capsid protein, which forms the outer viral shell. The putative capsid-encoding sequences identified by Kauffman and colleagues were most like those that encode a fold known as a double jelly roll. This fold was previously associated with nontailed viruses, in contrast to the HK97 fold found in tailed bacteriophages ${ }^{10}$.

To investigate the ecological role of autolykiviruses, the authors performed a monumental host-range analysis, testing the ability of their 241 marine-isolated viruses to infect and kill 318 of their marine bacterial strains. They found that the 18 non-tailed autolykiviruses were responsible for a disproportionately high number of bacterial cell deaths. This was mainly because the autolykiviruses had a substantially wider host range than the tailed group, and could infect multiple Vibrionaceae genera, whereas the tailed viruses studied could not. The authors therefore propose that the impact of autolykiviruses on the marine environment might be fundamentally 